Voix et Images

voixetimages

\title{
Les écrivaines dans l'histoire littéraire québécoise
}

Janine Boynard-Frot

Volume 7, numéro 1, automne 1981

Adrien Thério

URI : https://id.erudit.org/iderudit/200309ar

DOI : https://doi.org/10.7202/200309ar

Aller au sommaire du numéro

Éditeur(s)

Les Presses de l'Université du Québec

ISSN

0318-9201 (imprimé)

1705-933X (numérique)

Découvrir la revue

Citer cet article

Boynard-Frot, J. (1981). Les écrivaines dans l'histoire littéraire québécoise. Voix et Images, 7(1), 147-167. https://doi.org/10.7202/200309ar d'utilisation que vous pouvez consulter en ligne.

https://apropos.erudit.org/fr/usagers/politique-dutilisation/ 


\title{
Les écrivaines dans I'histoire littéraire québécoise *
}

\author{
par Janine Boynard-Frot
}

Les histoires, manuels ou précis de littérature et anthologies se présentent sous forme de corpus de textes dits littéraires et sont censés représenter le patrimoine littéraire d'une nation. Cet héritage est constitué d'objets isolés de la production générale et dotés d'une valeur symbolique spécifique par opposition aux autres productions textuelles qui sont affectées, elles, d'une valeur marchande et rejetées dans des sous-catégories de la littérature telles que la paralittérature, la contre-littérature, l'infra-littérature...

La littérature, que les pratiques traditionnelles de l'enseignement $\mathbf{n}$ 'interrogeaient jamais, pouvait, jusqu'à ces dernières années, prétendre à un statut de quasi autonomie fondé sur son prétendu caractère atemporel et anhistorique, socle élaboré et consolidé par l'ensemble'des pratiques critiques accréditant cette définition de la littérature qui serait constituée «d'un corpus d'cuvres de valeur qui s'imposent à l'attention du monde par des qualités de style exceptionnelles». Cette vision idéaliste d'une littérature détachée de toute contingence matérielle historique, telle qu'exprimée par Olivar Asselin préfaçant l'anthologie de Fournier, se retrouve, à des degrés variables, chez tous les auteurs de manuels ou histoires de la littérature, canadienne-française ou française du Québec, auteurs qui, notons-le en passant, ne sont pas évalués ici en tant qu'auteurs d'ouvrages mais bien en tant qu'éléments de rouages idéologiques.

Répétant la tautologie «la littérature c'est les grands textes», tous les historiens ou critiques de la littérature font état, implicitement ou explicitement, dans des préfaces ou introductions à leurs ouvrages, de leur singulière capacité de discernement, état privilégié, qui les induit et les fonde à sélectionner «les auteurs les plus représentatifs»(Baillargeon), "les chefs-d'œuvre les plus

\footnotetext{
* Ce texte a été donné en communication au congrès de l'ACFAS sous le titre «De la pratique critique des histoires de la littérature du Québec à l'égard d'un corpus d'écrivaines de l'entre-geux-guerres $»$.
} 
riches, les plus stimulants, les plus significatifs et les œuvres majeures les plus nourrissantes» (Bessette, Geslin, Parent), "les œuvres marquantes des meilleurs auteurs (Tougas), «des valeurs sûres ou ... des promesses éclatantes (Duhamel).

Chez Pierre de Grandpré l'approche se teinte d'un illusoire pragmatisme par le biais d'une feinte remise en question de l'arbitraire de la fonction sélective exercée par l'historien. "L'histoire, déclare cet auteur, n'est pas... si objective (puisqu'elle) repose à tout instant, ne serait-ce qu'en raison de ses choix et de ses exclusions sur l'appréciation critique" '. Mais la force de «l'habitus", pour reprendre l'expression de Bourdieu, a tôt fait d'infléchir le raisonnement critique ramené à des conceptions traditionnelles quant à la perception esthéthique des objets d'art, essentiellement fondée sur une «relation charismatique à l'art ", de sorte que l'arbitraire, loin d'être démasqué se trouve conforté par le discours de cet historien qui présente son activité comme «la mise en ordre chronologique en même temps qu'explicative d'une matière au sein de laquelle un historien, qui est de surcroît homme de goût, (nous soulignons) introduit constamment des jugements de valeur... jugements d'ordre esthétique (qui) allègent ou enrichissent, en tout cas modifient perpétuellement l'objet même de la recherche ${ }^{2}$.

Par la compétence artistique dont il s'investit, I'historien peut, sous l'effet d'un goût singulier, assimilable à un don ou à un état de grâce, "s'attacher, toujours selon P. de Grandpré, aux œuvres les plus hautes pour leur arracher leur secret de fabrication ou faire resplendir leur mystère » 3 . Ainsi dotée, sous l'effet d'une sacralisation de son propre rôle, d'un pouvoir indiscutable parce que d'essence quasi divine, la critique s'attribue-t-elle la fonction de légitimer et consacrer les objets qu'elle distingue.

Coupée de la réalité objective par la sublimation de sa pratique, de ses agents et des objets auxquels elle s'applique - les auteurs étant des génies et leurs productions des mystères - la littérature, située dans un ordre transcendantal, est ainsi soustraite au questionnement, toujours vulgaire, simpliste ou réductioniste, de ceux qui, non touchés par la grâce, analysent les textes non plus dans leur univers sacré et dans le respect des rites, mais à partir de ce qui les détermine dans l'Histoire et dans leur histoire.

Par ailleurs, des contradictions profondes agitent la pratique de l'historien qui nie la réalité objective et son caractère déterminant quand il s'agit d'appréhender les textes d'auteurs mais qui ancre délibérément sa propre production dans l'appareil scolaire, lieu matériel par excellence, et qui anticipe des effets non moins matériels de sa pratique. Citons, à ce propos, quelques remarques introductives contenues dans la préface du manuel des auteurs Bessette-Geslin-Parent,

La littérature, déclarent-ils, n'est pas un luxe. Bien au contraire, elle est une fonction... la littérature a pour fin de transformer les activités individuelles et sociales d'un groupe humain organisé en phénomènes de conscience nationale... L'école... a reçu de la Nation la mission privi- 
légiée de mener à bien... ce travail introductif de formation humaine... D'où le rôle éminent de l'enseignement littéraire dans la formation sociale et humaine de la conscience... (car) les textes littéraires sont des instruments d'élite de cette naissance de l'animal humain à la conscience humaine ${ }^{4}$.

Alors que l'ensemble des historiens tend à masquer le concept de fonction de la Littérature sous le camouflage de la gratuité de l'art, présenté comme une cause pour laquelle on se dévoue, puisque c'est toujours pour rendre service au maître et à l'élève, au professeur et à l'étudiant que l'on a entrepris ce travail laborieux qu'est l'histoire de la littérature, ces derniers auteurs eux, exhibent ce concept de fonction pour mieux le refouler, stratégie discursive créant l'illusion d'une perception artistique pragmatique mais dont la visée idéologique est de solidifier les bases de la convention institutionnelle par un raffermissement, dans un style «moderne», des conceptions traditionnelles selon lesquelles la littérature, garante des valeurs universelles, n'aurait d'autre fonction qu'humanisante.

Afin d'évaluer le rôle et la fonction, tant du manuel de littérature que du critique dans le champ social, nous nous proposons d'analyser le discours critique des différents auteurs de manuels ou d'histoires de la Littérature canadienne-française ou québécoise du Québec, de 1930 à 1970, et de repérer leurs fonctionnements dominants à partir du traitement discursif variable appliqué par ces différents auteurs à un corpus stable d'écrivaines de l'entredeux-guerres, soit Jovette-Alice Bernier, Alice Lemieux, Simone Routier, Eva Sénécal et Médjé Vézina dont les productions textuelles furent, à leur époque. légitimées et consacrées.

\section{Constitution du corpus}

Le corpus soumis à l'analyse est constitué des histoires littéraires ${ }^{5}$ qui ont en commun la production d'un discours sur la production littéraire de la période de l'entre-deux-guerres. Seule la fraction de discours concernant les écrivaines de l'époque concerne notre analyse, laquelle s'attache à la description du mode d'énonciation de ces discours, eux-mêmes déterminés par un ensemble de variables et invariants en rapport avec la situation de l'énonciation.

\subsection{Conditions de l'énonciation}

On devrait s'attendre, en raison des modifications qui ont marqué la formation sociale de 1930 à 1970 , à des disparités entre les perceptions artistiques de ces différents critiques qui ont été sous l'influence de modes de culturation fort différents, différences relatives à l'état de culture d'une époque donnée mais liées aussi au statut social de l'historien lequel est constitué d'un ensemble de déterminations allant de son degré de richesse lui donnant ou refusant l'accès à un certain type de culture, jusqu'à un ensemble 
de rapports sociaux humains qui sont autant de déterminations objectives influençant ses modes d'expression, ses représentations imagées du réel, la structuration même de son discours et l'émergence de ce discours dans I'Institution scolaire.

Cette analyse n'étant pas exhaustive, nous nous attacherons tout particulièrement aux aspects multiformes de ce discours mais rejetterons un ensemble d'éléments déterminant la situation d'énonciation. Par exemple, nous ne chercherons pas à savoir pourquoi l'Histoire de la littérature de Berthelot Brunet est, de l'avis de Paul Wyczynski ${ }^{6}$, "l'exemple typique de la façon dont il ne faut pas écrire l'histoire", jugement qui repousse cet ouvrage au bas de l'échelle de la légitimité, mais à quels effets de lecture cela aboutit quand les écrivaines de notre corpus sont impliquées dans le discours de cet historien, indépendamment donc des déterminations scolaires et autres qui ont pu orienter le projet de Brunet.

\subsection{Conditions d'apparition du corpus d'écrivaines dans les histoires littéraires}

Le manuel de littérature fait plus que consacrer l'arbitraire division entre littéraire et non littéraire: il reproduit la vision d'un monde hiérarchisé et la conforte en formant les mentalités des usagers de l'Institution scolaire à percevoir le réel et les humains dans des rapports hiérarchisés ce qui se réalise et se donne à lire dans un ensemble de procédés orientés, telles la présence ou l'absence de certains auteurs dans la table de matières, la mise en relief ou marginalisation des auteurs par l'utilisation de caractères typographiques particuliers (gras/majuscules vs italique/minuscules, etc...), les dispositions typographiques particulières (position en début ou fin de texte/ position dans le texte ou hors texte, par l'adjonction de catégories particulières, de notes en bas de page, etc.). On observe, en outre, l'introduction des auteurs dans des rapports de contiguïté avec d'autres auteurs (féminins/masculins, ou féminins/féminins), sans compter les citations présentes ou absentes/ absentes, ou dosées (de quelques mots à quelques lignes ou à quelques pages), soit tout un ensemble de marques métatextuelles aboutissant à l'effet de lecture recherché. 


\begin{tabular}{|c|c|c|c|c|c|c|c|c|c|c|c|}
\hline Histoires littéraires * & $\begin{array}{l}\text { C.R. } \\
1930\end{array}$ & $\begin{array}{c}\text { C.R. } \\
1939\end{array}$ & $\begin{array}{r}\text { Ste-A. } \\
1944\end{array}$ & $\begin{array}{l}\text { B.B. } \\
1946\end{array}$ & $\begin{array}{l}\text { A.V. } \\
1954\end{array}$ & $\begin{array}{c}\text { S.B. } \\
1957\end{array}$ & $\begin{array}{l}\text { G.T. } \\
1960\end{array}$ & $\begin{array}{l}\text { R.D. } \\
1967\end{array}$ & $\begin{array}{c}\text { P.deG. } \\
1968\end{array}$ & $\begin{array}{c}\text { B.G.P. } \\
1968\end{array}$ & $\begin{array}{l}\text { P.G. } \\
1969\end{array}$ \\
\hline 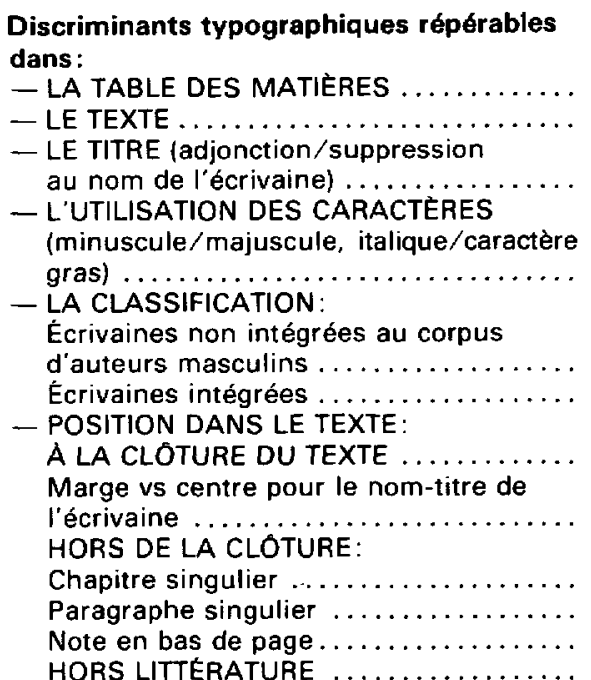 & (1) & $\begin{array}{l}0 \\
0 \\
\times\end{array}$ & $\begin{array}{l}0 \\
0\end{array}$ & $\bar{\emptyset}$ & $\begin{array}{l}\emptyset \\
0\end{array}$ & $\begin{array}{l}\bar{\emptyset} \\
x\end{array}$ & $\begin{array}{l}x \\
x\end{array}$ & 0 & $\begin{array}{l}x \\
x \\
x\end{array}$ & 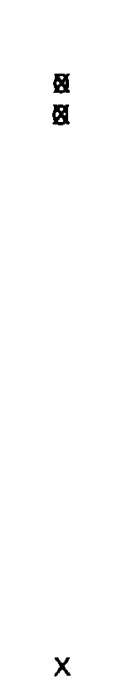 & 0 \\
\hline
\end{tabular}

Présence du corpus: entier $\ldots \ldots \ldots \ldots \ldots \ldots \ldots \ldots \ldots \ldots \ldots$

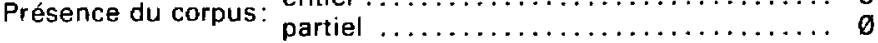

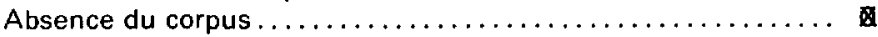

Absence de tout nom d'auteur dans la table des matières......

* Voir la liste des auteurs des histoires littéraires à la fin de l'article. 
Le relevé de ces pratiques est schématisé dans le Tableau I d'où nous dégageons les principales lignes de force suivantes:

$1^{\circ}$ Quatre histoires sur sept annoncent le corpus dans la table des matières, (dont trois datent de la période avant guerre) et sept histoires sur onze l'intègrent dans le texte.

$2^{\circ}$ Tous les historiens isolent la production féminine de la production masculine, sauf Brunet. Cela expliquerait, en partie tout au moins, le jugement sévère de $P$. Wyczynski sur le manuel de Brunet.

$3^{\circ}$ Tous refoulent cette production féminine aux confins de leur discours dans un HORS TEXTE (chapitre, paragraphe, note en bas de page) qui se lit HORS LITTÉRATURE, ce qui se réalise effectivement dans I'Histoire de Bessette-Geslin-Parent (1968), de même d'ailleurs que dans celle de Paul Gay (1969), qui se borne à citer quelques noms en bas de page sous un "NB".

Citons, à titre d'exemples, quelques titres de catégories discriminantes dans lesquelles sont isolées les femmes:

"Quelques poètes mineurs", (Duhamel)

«Femmes poètes", (C. Roy)

«Le romantisme féminin des années 30», (P. de Grandpré).

Ajoutons à cela l'effet discriminant des dispositions et caractères typographiques observé chez:

$P$. de Grandpré qui présente un auteur masculin par chapitre, pour la période étudiée, avec nom en titre vs toutes les femmes regroupées en un seul chapitre.

G. Tougas qui présente le nom des auteurs masculins au centre de la page, en majuscules vs nom des écrivaines en retrait et en minuscules.

C. Roy qui introduit une différenciation dans le mode d'interpellation. Pour les hommes, prénom + nom, ex.: Alfred DesRochers et pour les femmes, statut marital + prénom + nom, ex. : Melle Simone Routier.

A ces exemples il convient d'ajouter les effets de dérivations féminines, car exceptionnelle est I'utilisation de poétesse à qui est substitué "l'auteur féminin» ou le "poète».

Le tableau ci-joint révèle donc l'effet de marginalisation auquel conduit la pratique critique des différents historiens quand l'objet de leur discours est le corpus féminin. Cette pratique n'est évaluée que du point de vue du maniement idéologique des instruments objectifs tels que l'espace du livre ou les différents caractères d'écriture. II s'agit en fait de la phase élémentaire de la mise en place d'un dispositif de marginalisation dont l'efficacité de fonctionnement observé dans cette mise en scène du discours atteint son plein rendement au fur et à mesure que se déroulent les contenus. 


\section{Analyse du discours critique}

\subsection{Utilisation des éléments de morphòlogie sociale dans le processus de sacralisation de l'écrivaine}

Les histoires littéraires parsèment leurs discours de données biographiques sur les auteurs. Pêle-mêle, des informations sont données sur l'identité de l'auteur (date et lieu de naissance), sur ses origines, sa dotation culturelle, sa situation maritale, professionnelle, ses activités extra-littéraires, sa vie sentimentale, les distinctions: prix et médailles qui lui furent attribués. Ce magma d'informations varie, quantitativement et qualitativement, d'une Histoire à l'autre, créant, à la lecture, un effet de brouillage des conditions de production des textes littéraires et du statut de l'écrivain, déterminés, dans les faits, par des caractéristiques d'ordre social, et liés aussi au positionnement de l'écrivain dans le champ littéraire.

Parce que seule la lecture fonde le texte dans son existence, l'écrivain est contraint, par suite de l'absence de filières visant à le doter d'une formation spécifique qui déboucherait sur le métier d'écrivain, de se mettre en position de quête de la légitimité dans la sphère du littéraire qui se définit comme un système par son réseau d'instances et ses mécanismes de reproduction. L'émergence de l'écrivain est alors directement reliée aux stratégies qu'il élabore pour se faire connaître, à sa participation et à son adhésion à différents groupes ou cénacles, à ses relations inter-personnelles avec les éditeurs, critiques, journalistes, écrivains, etc., de même qu'à ses positions idéologiques, esthétiques.

Compte tenu du rôle déterminant, tant du système de cooptation dans le champ du littéraire, que de la dotation socio-culturelle de l'artiste, dans le processus de la consécration, il semble pertinent d'analyser les données biographiques sélectionnées par l'Histoire Littéraire de 1930 à 1970, pour chacune des écrivaines, et l'usage qu'elle en fait compte tenu de la biographie réelle des écrivaines. 
ÉLÉMENTS DE MORPHOLOGIE SOCIALE

TABLEAU ॥ SELLECTIONNÉS PAR LES HISTOIRES LITTÉRAIRES

\begin{tabular}{|c|c|c|c|c|c|c|c|c|c|c|}
\hline \multirow{2}{*}{$\begin{array}{l}\text { Écrivaines } \\
\text { BERNIER }\end{array}$} & \multicolumn{2}{|c|}{$\begin{array}{l}\text { Naissance } \\
\text { Date Lieu }\end{array}$} & \multirow{2}{*}{ Ascendance } & \multirow{2}{*}{$\begin{array}{c}\begin{array}{c}\text { Formation } \\
\text { scolaire }\end{array} \\
\end{array}$} & \multirow[t]{2}{*}{$\begin{array}{c}\text { Situation } \\
\text { profession. }\end{array}$} & \multirow{2}{*}{$\frac{\begin{array}{c}\text { Situation } \\
\text { maritale }\end{array}}{x}$} & \multirow[t]{2}{*}{$\begin{array}{l}\text { Adhésion } \\
\text { idéologique }\end{array}$} & \multirow[t]{2}{*}{ Stratégies } & \multicolumn{2}{|c|}{$\begin{array}{c}\text { Distinctions } \\
\text { Etrangères Canadiennes }\end{array}$} \\
\hline & $\begin{array}{l}x \\
x\end{array}$ & & & & & & & & & \\
\hline LEMIEUX & $\begin{array}{l}x \\
x\end{array}$ & & & & & $\begin{array}{l}x \\
x\end{array}$ & & & & \\
\hline ROUTIER & $\begin{array}{l}x \\
x \\
x \\
x\end{array}$ & $\begin{array}{l}x \\
x\end{array}$ & $x$ & $\begin{array}{l}x \\
x\end{array}$ & $\begin{array}{l}x \\
x \\
x\end{array}$ & $\begin{array}{l}x \\
x\end{array}$ & $\begin{array}{l}x \\
x \\
x \\
x \\
x \\
x\end{array}$ & $\begin{array}{l}x \\
x \\
x \\
x \\
x \\
x\end{array}$ & $\begin{array}{l}x \\
x \\
x \\
x \\
x\end{array}$ & $\begin{array}{l}x \\
x \\
x \\
x \\
x\end{array}$ \\
\hline SENECAL & $\begin{array}{l}x \\
x\end{array}$ & & & & & $x$ & & & $\begin{array}{l}x \\
x\end{array}$ & \\
\hline VÉZINA & $\begin{array}{l}x \\
x\end{array}$ & & & & & $x$ & & & & \\
\hline
\end{tabular}

Le nombre de marques « $X$ » correspond au nombre de fols que les données recherchées figurent dans le corpus des Histoires Littéraires envisagé. 
TABLEAU III

\begin{tabular}{|c|c|c|c|c|c|}
\hline \multirow[b]{2}{*}{ ECRIVAINES } & \multirow[b]{2}{*}{ DISTINCTIONS } & \multirow[b]{2}{*}{ AFFILIATIONS } & \multirow[b]{2}{*}{ PRODUCTIONS } & \multirow{2}{*}{\multicolumn{2}{|c|}{$\begin{array}{l}\text { FORMATION } \\
\text { PRIMAIRE } \\
\text { SECONDAIRE }\end{array}$}} \\
\hline & & & & & \\
\hline BERNIER & $\begin{array}{l}x \\
x \\
x\end{array}$ & $\begin{array}{l}x \\
x \\
x\end{array}$ & $\begin{array}{l}x \\
x \\
x \\
x \\
x \\
x \\
x\end{array}$ & $x$ & \\
\hline LEMIEUX & $\begin{array}{l}x \\
x\end{array}$ & $x$ & $\begin{array}{l}x \\
x \\
x\end{array}$ & & $x$ \\
\hline ROUTIER & $\begin{array}{l}x \\
x \\
x \\
x\end{array}$ & $\begin{array}{l}x \\
x \\
x \\
x\end{array}$ & $\begin{array}{l}x \\
x \\
x \\
x \\
x \\
x \\
x\end{array}$ & & $x$ \\
\hline SÉNÉCAL & $\begin{array}{l}x \\
x \\
x\end{array}$ & $x$ & $\begin{array}{l}X \\
X \\
X \\
X\end{array}$ & $x$ & \\
\hline VÉZINA & $\begin{array}{l}x \\
x \\
x \\
x\end{array}$ & & $x$ & & $x$ \\
\hline
\end{tabular}


Histoires G. Roy G. Roy Ste-Anne Brunet 1930 1939 1944 1946

A. Viatte Baillargeon G. Tougas Duhamel Grandpré 1954 1957 1960 1967

1968

B.G.P.

P. Gay

Ecrivaines

BERNIER

X

$x$

$x$

- - -

$\emptyset$

$\emptyset$

$-$

$-$

0

$\emptyset$

LEMIEUX

a

ROUTIER

X

$\mathrm{X}$

$x$

X

一

$\mathrm{X}$

$\emptyset$

SËNECAL

SĖNÉCAL

$+2$

$+2$

\section{VÉZINA}

$x \quad x$

$x$

$\mathrm{X}$

$\mathrm{X}$

Distinguée

Non distinguée $\ldots \ldots \ldots \ldots \ldots$.

Exclue $\ldots \ldots \ldots \ldots \ldots \ldots \ldots \ldots$ 
Du Tableau II, qui rend compte des résultats, se dégagent, dans un premier temps, les remarques préliminaires suivantes:

1) Routier polarise le plus grand pourcentage de marques. Bernier, Lemieux, Sénécal, Vézina n'apparaissent qu'en deux occasions: une fois au chapitre de la date de naissance et une autre fois indexées par leur statut marital: Madame étant opposée à Mademoiselle et si Alice Lemieux est remarquée par deux historiens c'est grâce à ce changement de statut de célibataire à mariée! II est presque inutile de préciser qu'il s'agit là d'une pratique discriminante observée à l'égard des femmes.

2) Outre Routier, Sénécal est la seule écrivaine du corpus dont le nom figure, à deux reprises, au chapitre des distinctions reçues.

Une recherche, visant à vérifier la conformité de ces données, qui créent un effet de hiérarchisation incontestable dans la mesure ou l'Histoire Littéraire représente une écrivaine polarisant l'ensemble des marques distinctives, démontre, ainsi que l'atteste le Tableau III, qu'au chapitre des distinctions, (prix, médailles et diplômes) et d'un point de vue quantitatif, Routier ne fut guère plus marquée que Bernier ou Sénécal. Par ailleurs, le prix David, dont i) est fait mention par certains historiens, a été partagé également entre Routier et Lemieux qui n'est citée, pour cette distinction, dans aucune histoire. Le processus de sacralisation de Routier implique donc la minorisation des autres éléments du groupe ce qui se réalise, dans l'Histoire Littéraire, par l'oblitération pure et simple, des faits. En ce qui concerne l'affiliation à différentes sociétés, cénacles ou institutions, la participation de Routier fut sensiblement équivalente à celle de Bernier, et en ce qui a trait à leurs productions, elles s'équilibrent, si nous considérons toujours le seul aspect quantitatif.

Les données biographiques, telles que sélectionnées par l'Histoire, visent à hiérarchiser le corpus et se trouvent dans un rapport d'intrication avec d'autres éléments du discours critique qui interviennent pour conforter cet effet. Il en est ainsi des jugements ou évaluations qui peuvent être directs ou médiatisés par d'autres jugements d'écrivains ou de critiques consacrés, jugements portant sur la personne de l'auteur, sur l'auteur ou sur "l'cuvre».

Parmi ces jugements qui visent à distinguer une ou plusieurs écrivaines du corpus, nous ne retiendrons, en vue de la constitution d'un tableau récapitulatif, que les jugements directs et excluons les quelques jugements indirects du type:

«rangeons-nous à l'avis de Monsieur Séraphin Marion, qui porte sur lui (L'Immortel Adolescent de Routier) ce jugement: "CEuvre remarquable par la technique etc...»?

Les commentaires évaluatifs visent les productrices et leurs productions sous deux aspects. La productrice est susceptible d'être évaluée en tant que créatrice dans l'ordre des créateurs: 
"Ce dernier recueil (Tout n'est pas dit de Bernier) place l'auteur au rang de nos meilleurs poètes" 8 .

ou en țant qu'être sexué, dans l'ordre des représentations que l'on se fait de la femme:

“Jovette-Alice Bernier, "plus femme que poète "...9"

"Éva Sénécal a chanté en des vers langoureux de jeune fille.. ${ }^{10}$ "

"Certaines affirmations étonnent sous la plume d'une jeune fille ${ }^{11}$ ".

De même, sa production littéraire est susceptible d'être évaluée (distinguée, non distinguée ou rejetée) sur la base de critères non spécifiés, ce qui atteste bien une relation charismatique à l'art.

“... il s'y (dans Les Tentations de Routier) rencontre de jolies pièces, mais ce n'est pas encore le chef- $d^{\prime}$ oeuvre (nous soulignons) ${ }^{12}$ „

"Son œuvre (Chaque Heure. a son visage de Vézina) est certainement originale, belle et forte... ${ }^{13}$ "

«Partie, du romantisme sentimental et ironique de quelques-unes de ses sœurs... sa poésie... (Routier) est bientôt parvenue à une assez extraordinaire concentration de l'intelligence et du sentiment... ${ }^{14}$ ”

ou encore explicitement jugée et cotée sur la base des positions idéologiques de l'autrice:

«Réponse à Désespoir de vieille fille (de Routier)... montre la foi la plus vraie que $j$ 'aie rencontrée... Cette poésie est plus discrète que celle de ses scurs... ${ }^{15}$ »

«En contraste, (nous soulignons) avec la cohorte des poétesses amoureuses de son temps, Simone Routier ne laisse jamais le sentiment dominer... ${ }^{16}$,

"Ses vers (Bernier) ne laissent filtrer aucun rayon d'espérance chrétienne, seule génératrice de joie et de paix ${ }^{17}$ \%.

De l'ensemble des cotes ainsi attribuées par les historiens, il découle ${ }^{18}$ que:

- La pratique évaluative se modifie radicalement après la guerre, les écrivains étant, à partir de cette période, moins appréhendées à titre de créatrices, comme le fait $C$. Roy, qu'à titre de femmes ${ }^{19}$.

- Routier n'est jamais exclue du corpus littéraire par les historiens qui incluent la production féminine dans leur Histoire tandis que les autres y sont exposées.

- Bernier et Vézina sont l'objet de jugements contradictoires. Leur production, légitimée avant 1945, tend à être occultée après cette date, tandis que Routier voit sa production légitimée à partir de cette période.

- Bernier et Vézina concentrent un pourcentage de marques évaluatives (positives ou négatives) qui les place en concurrence avec Routier, tandis qu'aucune marque évaluative n'est attribuée ni a Lemieux ni à Sénécal. Or dans le Tableau II synthétique des éléments de morphologie sociale sélectionnés par les Histoires Littéraires c'est 
Sénécal qui est, après Routier, la plus marquée et non pas Bernier ou Vézina.

Ces informations complémentaires orientent la lecture du Tableau II des données biographiques et favorisent la mise à jour de quelques mécanismes de fonctionnement de l'Histoire Littéraire, à savoir:

$1^{\circ}$ que I'Histoire Littéraire "canadienne-française» ou "française du Québec" retient, en priorité, au chapitre des distinctions, celles d'origine étrangère et accessoirement celles du pays, de sorte que Routier se trouve distinguée par une préface de Fernand Gregh, par un texte dédié à Jean Cassou, par son séjour à Paris, éléments de prestige soulignés par l'ensemble des historiens, ainsi que par un épigraphe de Paul Morin, plus associé à la culture française qu'à la culture québécoise * . Le Prix David et l'affiliation à l'Académie canadienne servent d'appoint mais ils n'entrent pas comme éléments déterminants dans le processus de sacralisation de l'écrivaine puisque, nous l'avons vérifié, I'Histoire peut omettre de fournir ces données (cas de Lemieux récipiendaire du Prix David). La présence arbitraire de Sénécal, qu'aucune Histoire ne distingue de ses consœurs par des jugements évaluatifs, s'explique donc par le fait qu'elle fut distinguée, au concours du Salon des Poètes de Lyon où elle remporta le premier prix de poésie en 1928, tandis que Bernier, qui ne reçut de distinctions que canadiennes et qui n'adhéra qu'à des cénacles québécois, est éliminée du chapitre des distinctions. La France, et Paris tout particulièrement, demeurent, dans l'idéologie littéraire, de 1930 à 1970 , le lieu dominant de la culture. Ce sont les instances de consécrations. Là est le pouvoir légitime de sorte qu'un texte, du seul fait de sa préface signée Fernand Gregh, a une tout autre valeur symbolique qu'un texte primé au concours d'Action Intellectuelle ou récipiendaire d'un prix Albert Levesque.

$2^{\circ}$ que I'Histoire Littéraire, reproduisant la dépendance culturelle à l'égard de la France par son processus d'élection (sélection et exclusion de certaines données) reflète des positions de classes, car ce n'est pas par hasard qu'elle fait état de la dotation culturelle de Routier (études supérieures), de son ascendance (petite nièce de Françoix-Xavier Garneau), de son statut professionnel (carrière dans la diplomatie), de son adhésion idéologique (retraite chez les Dominicaines) et qu'elle soit muette à propos de Bernier, Lemieux, Sénécal, Vézina, moins bien dotées sur le plan socio-culturel: cursus scolaire bref (niveaux primaire, secondaire pour Bernier, Sénécal), carrières dans les pages féminines des journaux, origines sociales humbles. Par ailleurs, que l'Histoire précise le lieu de naissance de Routier (Québec) mais s'abstienne d'indiquer celui de Bernier née à St-Fabien

\footnotetext{
* Les marques au chapitre des distinctions étrangères mises entre parenthèses, dans le Tableau II, font référence à Paul Morin et à son statut ambigu.
} 
de Rimouski, de Lemieux née à St-Michel de Bellechasse, de Sénécal née à La Patrie, laisse entrevoir un mécanisme du fonctionnement particulier de l'Histoire Littéraire qui minoriserait les littératures régionales éloignées des centres où sont concentrées les instances de diffusion, de production et de légitimité littéraire.

$3^{\circ}$ que, par un effet de censure idéologique, l'Histoire Littéraire consacre certaines valeurs et en élimine d'autres. Routier, ainsi que nous l'observons sur le Tableau II est fortement marquée sur le plan de l'adhésion idéologique (dimension religieuse). Dans l'ensemble, les historiens sont attentifs à signaler le virage religieux "d'une âme amenée au dépouillement intérieur par la force des événements et par la pente de son tempérament", selon la perception de Baillargeon, qui prend soin de n'intégrer dans son Histoire qu'une fraction de la production, et des éléments biographiques de l'écrivaine, celle qui est postérieure à son virage religieux. Une des causes de la marginalisation de Bernier ou de Vézina se déduit de commentaires qui mettent explicitement l'accent sur la contravention aux normes morales:

"Jovette-Alice Bernier, déclare Viatte, mêle l'amour charnel à l'amour divin»20.

"L'auteur (Jovette-Alice Bernier) a le talent poétique», concèdent les Scurs Ste-Anne bien que "ses vers ne laissent filtrer aucun rayon d'espérance chrétienne" mais, ajoutent-elles, "on aimerait à le voir s'exercer sur d'autres thèmes que l'amour" ${ }^{21 .}$

Quant à Vézina on reconnaît que son «œuvre est... originale, belle et forte" mais on déplore "l'atmosphère sensuelle et païenne" 22 .

Cette analyse des éléments de morphologie sociale démontre que I'Histoire Littéraire intervient dans le processus de reproduction des rapports sociaux dans la mesure où les éléments les moins bien dotés sur le plan socioculturel se retrouvent au bas de l'échelle de la légitimité. Par hypothèse nous posons que ce fonctionnement s'applique tant aux productions masculines que féminines mais qu'il a des effets plus visibles chez les femmes, qui constituent une classe dont la majorité des éléments sont économiquement démunis, et qui, par conséquent, en tant que classe dominée et exploitée doit nécessairement apparaître dans cette position dans les appareils idéologiques d'État. Une analyse du discours critique devrait vérifier cette dernière proposition.

\section{Structuration du discours critique}

C'est autour d'une charnière constituée d'oppositions révélatrices d'un mode d'appréhension des textes littéraires peu variable d'une histoire à l'autre. puisqu'il s'agit moins d'analyser que de juger, que s'articule le discours critique. La valeur, et par conséquent le mode de hiérarchisation des productions littéraires, prend appui sur des concepts et notions flous, jamais explicités et par le fatt même supposés acquis ou évidents par et pour ceux qui, soumis aux codes de lecture dans l'Institution scolaire, les reçoivent. 
Signalons quelques-unes de ces oppositions remarquées pour leur recurrence:

$\begin{array}{ll}\text { Thème, inspiration } & \text { vs art, style, expression } \\ \text { Obscurité } & \text { vs clarté } \\ \text { Subjectif } & \text { vs objectif } \\ \text { Effusion sentimentale } & \text { vs stoïcisme } \\ \text { Instinct } & \text { vs raison } \\ \text { Fadeur } & \text { vs virilité, vigueur } \\ \text { Révolte, élans } & \text { vs maturité } \\ \text { Labeur, artisanat } & \text { vs spontanéité, artiste } \\ \text { Traditionnel } & \text { vs novateur, etc... }\end{array}$

Ces notions sont instables et sujettes à variations, dans leur application à un même objet, d'une histoire à l'autre.

Là ou C. Roy perçoit un manque de "clarté» de «spontanéité» et la marque du «labeur» (chez Routier), P. de Grandpré retient des «intuitions saisissantes». Là ou certains, entre autres G. Tougas, voient une régression, un «recul», dans "l'art» de Routier, au moment de la parution de «Psaumes au Jardin clos", d'autres, à l'exemple de P. de Grandpré, voient une envolée sous l'effet de la «lucidité et fermeté (qui) haussent le poème...».

Les contradictions affleurent au sein d'un même discours. On affirme, à propos de Sénécal : «à tout instant l'esprit est là qui dirige l'effusion " et dans le même souffle on met au compte «d'une involontaire hardiesse ", 23 donc hors du contrôle de l'esprit, un poème qui fut l'objet d'une distinction étrangère. Un élément qui devait logiquement soutenir une assertion est arbitrairement détourné de son parcours logique, sans explication. On pose, sans tenir compte des conditions matérielles de production, que le hasard est I'élément déterminant dans la production de l'écrivaine. Or les contraintes qui pesaient sur Sénécal, écrivant au Québec, dans un état de censure particulier, vers 1930. se levaient dès l'instant où cette production était destinée à la France. Seul ce facteur objectif explique la "hardiesse» du poème.

Ces notions dichotomiques, qui créent l'illusion d'une approche méthodique des textes littéraires ne peuvent constituer une base sûre d'analyse dans la recherche de ce qui fonde aux yeux de la critique la valeur des productions textuelles féminines.

L'objet poésie, de même que l'objet roman psychologique, est perçu, par l'ensemble des historiens critiques dans son rapport a un ensemble de règles censées régir les niveaux linguistiques et esthétiques mais ce qui polarise et constitue l'essentiel du discours critique c'est le principe de modératıon auquel devrait être tenu de se soumettre la créatrice. On reprochera aux écrivaines leur excès "d’éloquence», leur manque de «discrétion", leurs "effusions sentimentales", leur poésie * absolument subjective» et sur ce point. la critique est unanime pour manifester la non soumission des femmes à ce principe et leur tendance à l'immodération/l'immodestie/l'immoralité. 


\subsection{Métalangages}

Ces procédures de marginalisation de la poésie féminine, qui prennent appui sur des systématiques débordant la pratique critique des historiens, opèrent à différents niveaux du texte appréhendé au moyen de codes littéraires esthétiques, psychologiques.

Les codes littéraires. Le discours critique classe les productions selon les genres, les époques ou les écoles. Les classifications et catégories ainsi constituées sont prétendument basées sur des critères objectifs. Or, les productions des femmes n'entrent dans ces catégories, tels le lyrisme ou le romantisme, par exemple, qu'assorties d'une caractérisation qui les discrimine. Observons ce fonctionnement dans quelques exemples, notamment chez C. Roy qui, dans le jugement qu'il porte sur Vézina, manifeste tout d'abord une restriction suivie d'une levée de cette restriction:

«elle s'est exprimée, dit-il dans quelques-uns des meilleurs vers que nous ait donné le lyrisme féminin, le lyrisme tout court», (nous soulignons\} ${ }^{24}$.

Évaluons encore les contorsions d'un discours, chez P. de Grandpré, qui refoule les productions féminines hors du romantisme, dans un romantisme que l'on spécifie de «féminin" puis de "néo-romantisme», et de «postromantisme", autant de qualifications qui disqualifient une production rejetée dans une catégorie inférieure, un sous-romantisme assorti d'une définition singulière: poésie de nostalgie et d'aspirations vagues, de touffeurs, de complaisances à soi-même.... 25 .

S'appuyant sur le réseau de références et de légitimation mis en place par les instances et appareils idéologiques de la classe dominante, l'histoire littéraire fonde ses sélections sur des rapports qu'elle construit entre les productions sacralisées du passé et celles du présent. Comparer Routier à Racine comme le font Brunet et Baillargeon vise à la promouvoir dans la hiérarchie de la légitimité par la référence au style masculin noble, classique, etc..., tandis que comparer Bernier ou Vézina à la comtesse de Noailles ou à Renée Vivien qui furent traitées par Maurras, de "bacchantes», "d'escadron d'amazones», de "Métèques», «d'insurgées»26, contribue à maintenir ces dernières au bas de la hiérarchie.

Les codes esthétiques. Les codes esthétiques qui projettent des notions évaluatives sur des formes précises révèlent, dans leur fonctionnement, l'influence déterminante des préconstruits idéologiques qui les sous-tendent et qui représentent la «féminité». On retiendra des productions féminines "un art... plus délicat... plus joli... ${ }^{27}$, on sera sensible à la *jolie précision du vers» 28 , à la «tendresse rêveuse et délicate» 29 , à la «délicatesse féminine» 30 , à la candeur... à la charmante fantaisie... aux intuitions saisissantes... " ${ }^{31}$, etc. 
écrivaines ou de leur travail, révèlent un autre aspect discriminant de la pratique critique des histoires littéraires à l'égard des femmes que I'on qualifie de "faunesse déchaînée " ${ }^{32}$, de «bacchante enivrée» ${ }^{33}$, de "cohorte de poétesses amoureuses»34. de "peloton de 9 ou 10 poétesses»35. Ces désignations ne sont pas neutres. Elles connotent, par les sèmes qui les sous-tendent, la sauvagerie, la bestialité, le dérèglement des mœurs, de même que la multitude (par cohorte et peloton) qui se lit, à la fois dans son opposition à élite et dans son sens de troupe, populace, connotant la débauche et la vulgarité.

Un autre aspect du code, analogue dans son fonctionnement réducteur, se découvre dans l'attribution de qualifications prétendûment positives à la personne qui est alors substituée à son travail, ce qu'illustre cette évaluation laconique de Tougas:

II n'y a pas lieu, déclare-t-il, de revenir sur le verdict de Robert Choquette qui a qualifié Alice Lemieux d'«aimable poète" ${ }^{36}$.

ou encore ce commentaire de P. de Grandpré qui introduit Bernier en ces termes:

Avec Jovette-Alice Bernier, c'est autre chose. La tendre mutine et spirituelle Jovette des journaux, de la radio et des revues... ${ }^{37}$.

La mise en relief de la dimension affective de Bernier, associée à son activité para-littéraire, elle aussi mise en relief, crée l'effet de lecture recherché: Bernier est charmante mais sa production littéraire est secondaire.

A ce stade-ci de l'analyse, nous ne pouvons que reprendre les observations de Houbedine qui avance que «la langue n'est... pas ce lieu innocent de l'échange communicatif mais une indexation, sexuelle et sociale, discriminante, symptôme certes de la structure sociale, mais contribuant aussi à perpétuer ses discriminations voire à les renforcer» 38 .

\subsection{Théories}

Le discours critique s'élabore sur la base d'une conception des pratiques artistiques littéraires. On soumet que la «forme», par opposition au «fond», a valeur prééminente et qu'elle suppose l'acquisition de techniques et de connaissances.

"Imiter les modernes, de Baudelaire à Cocteau...", suggère-t-on ${ }^{39}$.

«(Simone Routier) s'était pliée à la dure gymnastique du vers traditionnel» rappelle-t-on ${ }^{40}$.

«Il lui (A. Lemieux) arrive d'employer des expressions incohérentes, obscures, d'aller même jusqu'à l'incorrection grammaticale", reproche-ton à l'écrivaine ${ }^{41}$.

L'ensemble des techniques ainsi suggérées constitue un savoir-faire codé et retransmis par et dans l'Institution scolaire. D'autres techniques ou connaissances visant l'acquisition d'un savoir-être fixant les normes du com- 
portement créateur et présupposant tout savoir-faire, semblent aux yeux des Historiens fonder en valeur toute poésie.

En dépit de leur imprécision des notions telles que la virilité ou la raison, constitutives de ces normes, jouent un rôle déterminant dans l'évaluation du savoir-faire de l'écrivaine.

On jugera Bernier à:

«Sa poésie, moins langoureuse ou plus virile que celle de Lemieux (et qui se pénètre d'un stoïcisme et parfois d'une ironie qui enlèvent au sentiment ce qu'il po,urrait avoir de trop fade ${ }^{42}$.

On distinguera Simone Routier qui :

«En contraste avec la cohorte... ne laisse jamais le sentiment dominer... ${ }^{43}$

ou qui est «...parvenue à une extraordinaire concentration de l'intelligence et du sentiment $\$ 44$.

Vont dans le même sens les remarques touchant le manque de «discipline» de "vigueur» de "discrétion« ou "l'excès d'éloquence» des poétesses qui traduisent une non adéquation entre la représentation que les femmes donnent «du" poète et celle préconstruite par l'idéologie dominante masculine.

On soumet, en définitive, que la norme du comportement qui détermine la reconnaissance du savoir-faire de l'écrivaine par l'Histoire Littéraire, c'est l'adéquation entre Femme et être désincarné, chirurgie critique par laquelle s'opère la métamorphose de la Femme en un Ange digne de figurer dans la littérature:

"Simone Routier, une âme intérieure et dépouillée», titre Baillargeon.

Tandis que Bernier, "la faunesse déchaînée», "la bacchante enivrée", qui ne se prête pas à une telle réduction, heurte l'idéologie littéraire:

"On est surpris et quelque peu mal à l'aise de la voir mettre ainsi son âme à nu devant le lecteur» s'offusquent les Sœurs de Ste-Anne qui concluent «La sensualité et la passion y trouvent leur compte et certaines affirmations étonnent sous la plume d'une jeune fille " ${ }^{45}$.

Toute inadéquation entre femme et «âme" est dès lors imputable à un manque de savoir-faire que $P$. de Grandpré traduit en ces termes:

Ce peloton de neuf ou dix poétesses... dépassant de loin, non sans doute encore par l'art, (nous soulignons) mais par une intrépidité dans l'aveu de soi-même... leurs confrères masculins ${ }^{46}$.

On soumet que le texte poétique a pour fonction exclusive de représenter le monde, qu'il est chant, musique, peinture, qu'il produit du plaisir visuel, auditif, gustatif par les vers qui donnent une «note juste» les «accents déchirants», les poètes qui «chantent » et les «peintures fraîches et gracieuses" 
ou les «poèmes faciles à goûter » qu'ils produisent. Le texte n'est que transparence à l'auteur, et non transformation du réel, ce qu'exprime, on ne peut mieux, Baillargeon:

"Sa forme littéraire, toute diaphane, laisse transparaître l'âme", écrit-il à propos de Routier ${ }^{47}$.

De cette recherche, nous pouvons donc conclure que:

- Par son rôle d'éducateur dans le champ de l'Institution Scolaire, l'École étant précisément l'Appareil idéologique d'État qui, selon Althusser, joue le rôle dominant, l'Histoire Littéraire opère la socialisation des individus par l'inculcation de normes et valeurs ce qui lui confère une fonction légitimante et un rôle structurateur.

- Que cette socialisation implique un camouflage des intérêts de la classe dominante, masqués en valeurs.

- Que parmi ces intérêts la reproduction des rapports sociaux ou la division sociale en exploiteurs vs exploités est l'objectif visé par la classe dominante.

- Que dans l'ordre social les femmes entretiennent des rapports de soumission et de dépendance (économiques et politiques) face aux hommes, rapports dont la forme est retransmise par et dans l'appareil scolaire, par et dans la littérature, par et dans l'Histoire Littéraire.

- Que cette retransmission ne s'opère qu'au prix d'un détournement de savoir soustrait aux usagers de l'Appareil scolaire, ignorance du savoir sur la production réelle du travail littéraire qui se répercute, par la lecture/écriture, usque dans les œuvres du présent.

- Que la production réelle du travail littéraire des écrivaines de l'entredeux-guerres, par son pouvoir de transformation du réel, va à l'encontre du projet éducatif chrétien du système scolaire constitué d'un ensemble de règles et normes régissant les rapports interindividuels conçus dans la hiérarchie et immuables.

- Qu'en conséquence, et pour faire suite aux remarques de Jacques Duboıs qui observe que «le discours féminin en ce qu'il a de spécifique a "toujours) été barré en littérature comme ailleurs» 48 , il appartient aux femmes ie percevoir les rapports langue/discrimination sociale/discrimination sexuel' $\Leftrightarrow$. de mettre à jour les refoulés, certes, mais plus encore, d'intervenir sur la rangive en vue d'une transformation sociale et idéologique. 


\section{AUTEURS DES HISTOIRES LITTERAIRES}

Mgr Camille Roy, Histoire de la litterrature canadienne, Québec, Imprimerie de I'Action Sociale Itée, 1930.

Mgr Camille Roy. Manuel d'histoire de la littérature canadienne de langue française. Montréal, Librairie Beauchemin Ltée, 1939.

Les Sœurs de Sainte-Anne, Histoire des littératures française et canadienne, Lachine. Procure des Missions, 1944.

Berthelot Brunet, Histoire de la littérature canadienne-française, Montréal, Éditions de l'Arbre, 1946.

Auguste Viatte, Histoire littéraire de l'Amérique française des origines à 1950, Québec, Presses de l'université Laval, 1954.

Samuel Baillargeon, Littérature canadienne-française, Montréal, Fidès, 1957.

Gérard Tougas, Histoire de la littérature canadienne-française, Paris, Presses Universitaires de France, 1960.

Roger Duhamel. Manuel de littérature canadienne-française, Ottawa, Éditions du Renouveau Pédagogique Inc., 1967.

Pıerre de Grandpré, Histoire de la littérature française du Québec, Tome I et II, Montréal, Librairie Beauchemin Limitée, 1968.

Gérard Bessette, Lucien Geslin, Charles Parent, Histoire de la littérature canadiennefrançaise, Centre éducatif et culturel, Inc., 1968.

Paul Gay, Guide littéraire du Canada français, Montréal, HMH, 1969.

1. Pierre de Grandpré, Tome I, p. 7.

2. Id., ibid., p. 7.

3. Id., ibid., p. 12.

4. Bessette, Geslin, Parent, Préface, pp. 5 à 11.

5. Auteurs des histoires littéraires (Consulter Annexe $V$ )

C. Roy (éd. 1930, éd. 1939), Sœurs de Sainte-Anne (1944), B. Brunet (1946), A. Viatte (1954), S. Baillargeon (1957), G. Tougas (1960), R. Duhamel (1967), P. de Grandpré (1968), Bessette-Geslin-Parent (1968), Gay (1969).

6. In Littérature et société canadiennes-françaises, Québec, P.U.L. 1964, p. 23.

7. Les Sœurs de Sainte-Anne, p. 491.

8. Camille Roy, (1930), p. 181.

9. Viatte, p. 187.

10. Tougas, p. 114.

11. Sœurs de Sainte-Anne, p. 492.

12. Scurs de Sainte-Anne, p. 492.

13. Sceurs de Sainte-Anne, p. 493.

14. P. de Grandpré, p. 243.

15. Brunet, p. 88.

16. Baillargeon, p. 217.

17. Sceurs de Sainte-Anne, p. 492.

18. Tableau IV.

19. Chez P. de Grandpré, notamment, les productions émanent de la jeune fille langoureuse, de la "personnalité vigoureuse» de la femme. Ou encore, c'est l'émouvante production de la femme «mutine et spirituelle». 
20. Viatte, p. 187.

21. Sceurs de Sainte-Anne, p. 492.

22. Sœurs de Sainte-Anne, p. 493.

23. P. de Grandpré, p. 237.

24. C. Roy, éd. 1939, p. 120.

25. P. de Grandpré, p. 236.

26. Charles Maurras, L'Avenir de l'intelligence, Paris, Albert Fontemoing, 1905.

27. C. Roy, p. 180, (éd. 1930).

28. Tougas, p. 114.

29. Grandpré, p. 242.

30. Baillargeon, p. 218.

31. Grandpré, pp. 237 et 242.

32. Brunet, (de Bernier), p. 86.

33. Duhamel, (de Bernier), p. 70.

34. Baillargeon, p. 217 et Viatte, p. 187.

35. P. de Grandpré, p. 236.

36. Tougas, p. 114.

37. Grandpré, p. 260.

38. Houdebine A.M., Tel Quel, 1977, N 74, p. 91.

39. Viatte, p. 188.

40. Baillargeon, p. 218.

41. Soeurs de Sainte-Anne, p. 493.

42. C. Roy, (éd. 1930), p. 181

43. Baillargeon, p. 217.

44. P. de Grandpré, p. 243.

45. Sours de Sainte-Anne, p. 492.

46. P. de Grandpré, p. 236.

47. Baillargeon, p. 218.

48. Jacques Dubois, L'Institution de la littérature, Fernand Nathan/Éditions Labor, 1978, p. 134. 